\title{
Caracterización de Revestimientos de Niobio formados por Aspersión Térmica a Plasma sobre Acero API 5I X65
}

\author{
José F. Matos ${ }^{(1)}$, Felipe P. Motta ${ }^{(1)}$, Ester S. Rieder ${ }^{(2)}$ y Célia F. Malfatti ${ }^{(1)}$ \\ (1) Universidade Federal do Rio Grande do Sul, Escuela de Ingeniería, Departamento de \\ Metalurgia. (e-mail: célia.malfatti@ufrgs.br) \\ (2) Universidade Luterana do Brasil, Centro de Investigación en Materiales. \\ (e-mail: esterrieder@terra.com.br)
}

Recibido Sep. 28, 2011; Aceptado Nov. 22, 2011; Versión final recibida Ene. 09, 2012

\section{Resumen}

Se evaluó la morfología y el comportamiento electroquímico del revestimiento de niobio obtenido por el proceso de aspersión térmica a plasma en substrato de acero API 5L X65. El revestimiento con niobio se realizó con una corriente eléctrica de $500 \mathrm{~A}$, flujo de polvo metálico de niobio de 15 $\mathrm{g} / \mathrm{min}$ y gas de argón de $2.0 \mathrm{~L} / \mathrm{min}$. La preparación superficial del substrato se realiza con precalentamiento y limpieza a presión con abrasivo de alúmina. La morfología del revestimiento fue analizada por microscopía electrónica de barrido (MEB), la rugosidad de la superficie se evaluó por perfilometría, la dureza por micro-dureza Vickers y la adherencia por ensayado de doblado a $180^{\circ}$. La resistencia a la corrosión del revestimiento se determinó mediante el ensayo de polarización en una solución de $\mathrm{NaCl} 3,5 \%$. A partir de los resultados obtenidos fue posible verificar que el revestimiento presentó fisuras y poros entre las placas rociadas además de una dureza elevada en relación al substrato y adherencia no adecuada. Aún así los valores de densidad de corriente desarrollados por el sistema en comparación al acero revestido se redujeron.

\section{Characterization of Niobium Coatings obtained by Plasma Spray Thermal Substrate on Steel API 5I X651}

\begin{abstract}
The morphology and electrochemical behavior of niobium coating obtained by thermal plasma spray process on a substrate of steel API $5 \mathrm{~L}$ X65 was evaluated. The coating was obtained, using electrical current of $500 \mathrm{~A}, 15 \mathrm{~g} / \mathrm{min}$ of powder and $2.0 \mathrm{~L} / \mathrm{min}$ of argon gas. The preparation of the substrate surface was performed with preheating and abrasive blasting with alumina. After thermal spraying, the morphology of the coating was analyzed by scanning electron microscopy (SEM), while the surface roughness was evaluated by profilometry, hardness by Vickers microhardness test and adhesion by bending at $180^{\circ}$. The corrosion resistance of the coating was evaluated from the polarization test in $3.5 \% \mathrm{NaCl}$ solution. The results show that the coating presented cracking and lack of padding between the plates sprayed, relative high hardness and deficient adhesion to the substrate. However, the values of current density carried by the system compared to uncoated steel were reduced.
\end{abstract}




\section{INTRODUCCION}

La selección de revestimientos para aplicación en condiciones comúnmente presentes en la industria petroquímica, requiere una rigurosa especificación de requisitos a ser atendidos. La presencia de temperaturas y presiones elevadas además de fluidos altamente corrosivos exigen revestimientos con desempeños adecuados para que resistan en estos medios (Fenker, 2011).

La aplicación de revestimientos por aspersión térmica de niobio sobre substrato de acero al carbono viene surgiendo como una alternativa a los procesos de obtención de revestimientos por soldadura, soldadura en frío o en el estado sólido. (Majumdar, 2006; Zhang, 2008) Pocas investigaciones específicas en esta área fueron desarrolladas, demandando un mayor conocimiento sobre el tipo de proceso de aspersión térmica a plasma de arco no transferido y su relación con las propiedades de estos revestimientos.

El interés por la aplicación de niobio se debe a la posibilidad de obtener una película adherente y principalmente resistente a la corrosión, cuando se obtiene por de aspersión térmica El niobio forma una capa pasiva estable y espontanea $\mathrm{Nb}_{2} \mathrm{O}_{5}$ al aire, siendo resistente en un amplio rango de variación de $\mathrm{pH}$ y potenciales. Además de eso presenta una buena resistencia en medios oxidantes y reductores (Ardelean, 2008; Quelhas, 2007).

Estudios realizados mostraron que el revestimiento de niobio no sufre corrosión por picadura en medio aireado en solución de $\mathrm{NaCl} 3,5 \%$, ese comportamiento es superior al acero 316 bajo las mismas condiciones de ensayo (Hsieh, 1991).

En investigaciones realizadas fue analizado el comportamiento del niobio frente a la corrosión naftenica, utilizando ensayos en autoclaves para verificar la resistencia del niobio metálico en petróleo con IAT (índice de acidez total) igual a $1,33 \mathrm{mg} \mathrm{KOH} / \mathrm{g}$. Las tasas de corrosión encontradas fueron muy inferiores a aquellas encontradas en los aceros inoxidables que generalmente en operaciones que involucran petróleo (Carvalho, 2004). Una restricción para la aplicación de revestimientos de niobio puro es la temperatura de operación que debe limitarse a $300^{\circ} \mathrm{C}$, debido a que el niobio se deteriora con el oxigeno, hidrogeno y nitrógeno (Chaves, 2008). El niobio por ser un metal más noble que el substrato de acero requiere un control más riguroso en los procesos de aspersión térmica, en lo referente a la formación de defectos como la porosidad, por la formación de pilas galvánicas desfavorables entres estos metales. (Gentil, 1996; Carvalho 1998)

En relación a la calidad de los revestimientos que se obtienen por aspersión térmica, las tensiones residuales ejercen una influencia importante sobre el desempeño de estos revestimientos. Se puede destacar dos fuentes que influyen fuertemente sobre esas tensiones. La primera está relacionada con el enfriamiento brusco que sufren las partículas depositadas, desde la temperatura máxima hasta la temperatura que tiene, cuando choca contra el substrato. La segunda está relacionada con la diferencia del coeficiente de expansión térmica entre el material de recubrimiento y el material del substrato (Thorpe, 1993).

La corriente que se utiliza en el proceso influye en el calentamiento de las partículas de polvo y en la fusión de las mismas durante el recorrido desde la boquilla hasta el substrato (Paredes, 2004). La tasa de flujo del gas (argón e hidrogeno) influye sobre la proporción del polvo que se deposita y afecta la composición y calidad del revestimiento (Godoy, 2007). En este contexto, el objetivo del presente trabajo fue obtener revestimientos de niobio por el proceso de aspersión térmica a plasma y caracterizar esos revestimientos respecto a su comportamiento electroquímico.

\section{MATERIALES Y MÉTODOS}

Los revestimientos fueron obtenidos a partir del polvo de niobio con granulometría media de 23 $\mu \mathrm{m}$. Las partículas fueron previamente secadas en una estufa a una temperatura aproximada de $80^{\circ} \mathrm{C}$. 
La limpieza superficial del substrato se realizó con acetona, luego, un granallado con abrasivo de alúmina hasta obtener un acero áspero (superficie rugosa) y pre-calentado con un soplete hasta una temperatura de $120^{\circ} \mathrm{C}$ (monitoreado por un pirómetro).

La aspersión térmica se realizó en un equipo de plasma spray Sulzer Metco (TriplexPro ${ }^{\mathrm{TM}}$-200) (figura 1). Los parámetros de proceso fueron corriente eléctrica $500 \mathrm{~A}$, distancia entre el substrato y el soplete fue $10 \mathrm{~cm}$, flujo de polvo de niobio $15 \mathrm{~g} / \mathrm{min}$ y flujo de gas argón de 2,0 L/min. Se utilizó una pequeña cantidad de hidrógeno en el gas de ionización para complementar la energía de ionización del sistema. Las dimensiones del substrato fueron 120x20x11,5 mm, mecanizadas en el sentido longitudinal de un tubo sin costura (acero API 5L X65).

La morfología de la superficie y la sección transversal, del revestimiento con niobio y del acero áspero fue evaluada por microscopia electrónica de barrido (JEOL-JSM 5800 Scanning Microscope) con tensión de aceleración de $20 \mathrm{keV}$. También se evaluó la rugosidad de las muestras por perfilometría, en un perfilómetro (CETR PRO500 3D. Profilometer) y la micro-dureza Vickers del revestimiento en la sección transversal con un tiempo de 15 S y $200 \mathrm{~g}$ de carga.

La resistencia a la corrosión de los revestimientos se determinó a partir de curvas de polarización potenciodinámicas en solución de $\mathrm{NaCl} 3,5 \%$ empleándose para esto un potenciostato (PAR 273A) y una celda de tres electrodos (electrodo de referencia, calomelano y contra-electrodo de platino). La adherencia del sistema substrato-revestimiento fue evaluada por ensayo de doblado en un equipo MTS Servohydraulic de $100 \mathrm{KN}$ y el resultado se comparó con la norma PETROBRAS N-2568.
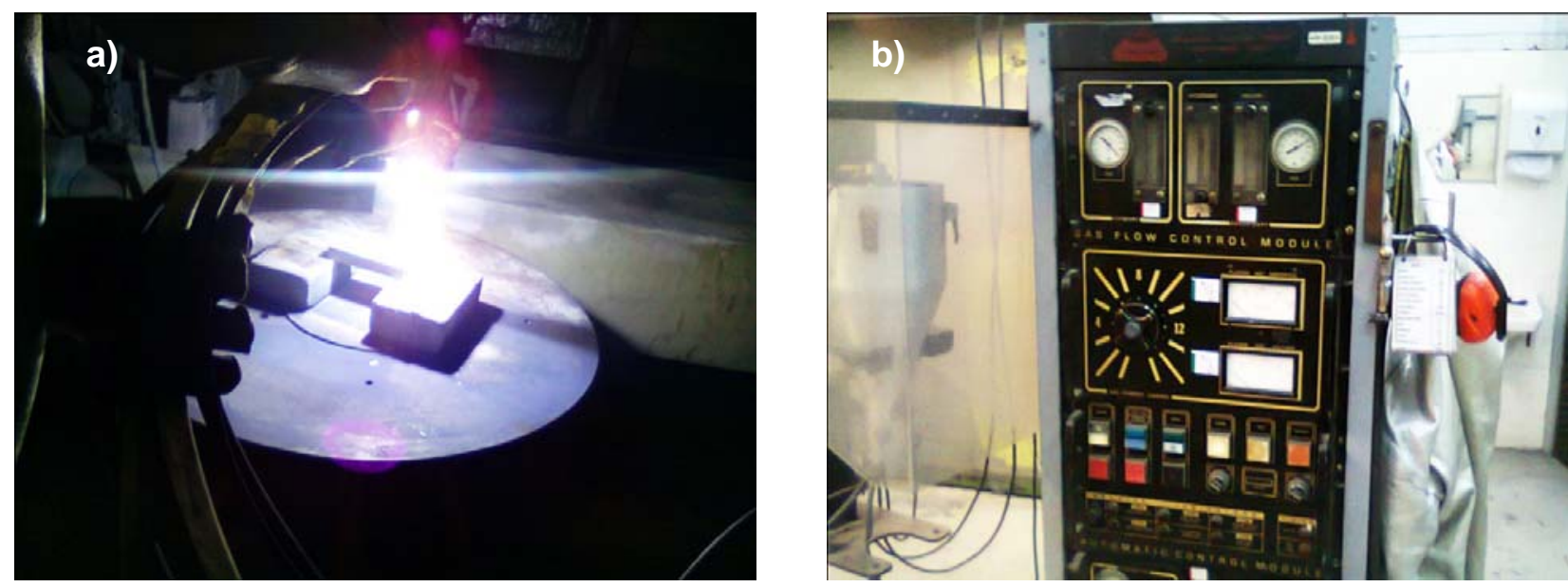

Fig. 1: Proceso de aspersión térmica a plasma a) Pistola de aspersión térmica a plasma, b) Equipo de control de parámetros operacionales del proceso

\section{RESULTADOS Y DISCUSIONES}

Una de las características más importantes de los revestimientos por aspersión térmica es la adhesión y cohesión de las partículas, siendo ella influenciada por diversos parámetros tales como la preparación superficial del substrato y la aspersión térmica.

La formación y composición de los revestimientos por aspersión térmica (figura 2), dependen principalmente de la temperatura del proceso, de la velocidad de salida y de los tipos de gases empleados en el transporte de las partículas atomizadas (Lima, 2001)

Es posible observar (figura 3) a partir de la imagen obtenida del corte transversal, que el revestimiento presenta vacios y falta de llenado entre las capas esparcidas con niobio la presencia de esos vacios puede estar relacionada (en parte) a la baja corriente eléctrica empleada en el proceso. Eso puede haber contribuido a que las partículas no lleguen a la temperatura adecuada y tengan una buena dispersión uniforme sobre el substrato. 


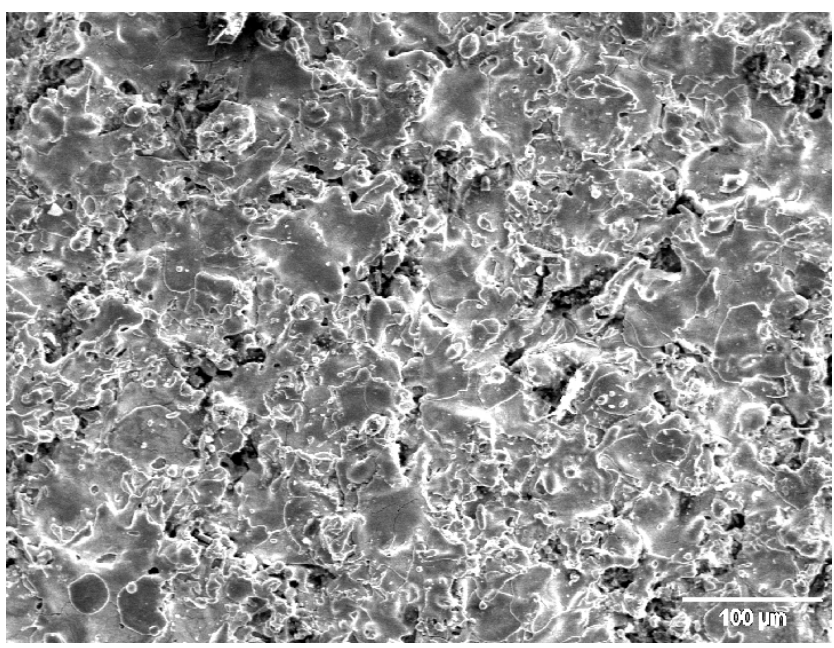

Fig. 2: Micrografías obtenidas por MEB del revestimiento de niobio

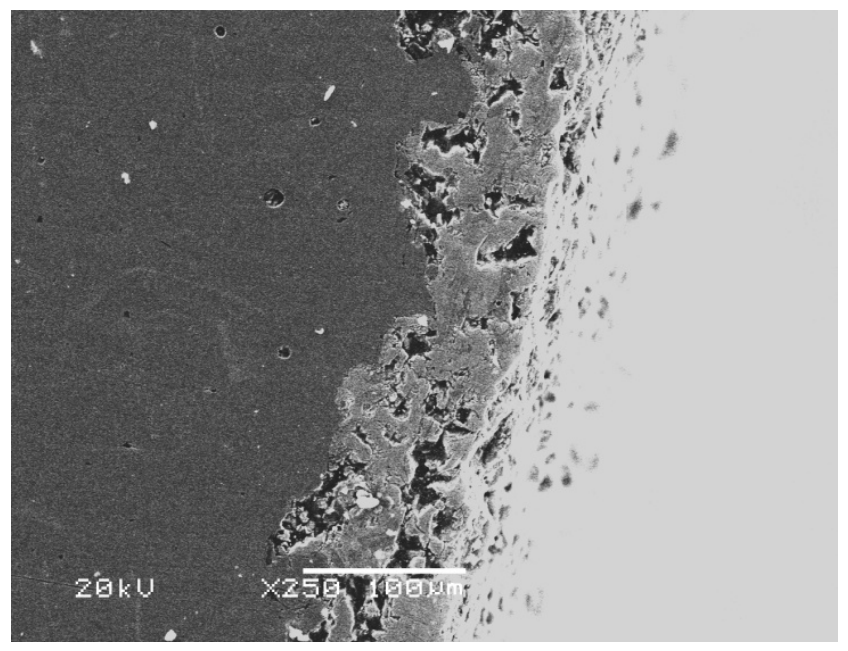

Fig. 3: Micrografía transversal del revestimiento por MEB

La rugosidad de la superficie del acero áspero influye en la adhesión del revestimiento al substrato, conforme la norma de la Petrobras N-2568 (Petrobras, 2004) que recomienda que la rugosidad superficial pulida debe de estar entre 75 y $120 \mu \mathrm{m}$.

A partir del análisis por perfilometría (figura 4) fue posible determinar el valor de Ry próximo a 60 $\mu \mathrm{m}$ para el acero áspero. El aspecto de la superficie de este acero puede ser observado en una imagen transversal (figura 5).

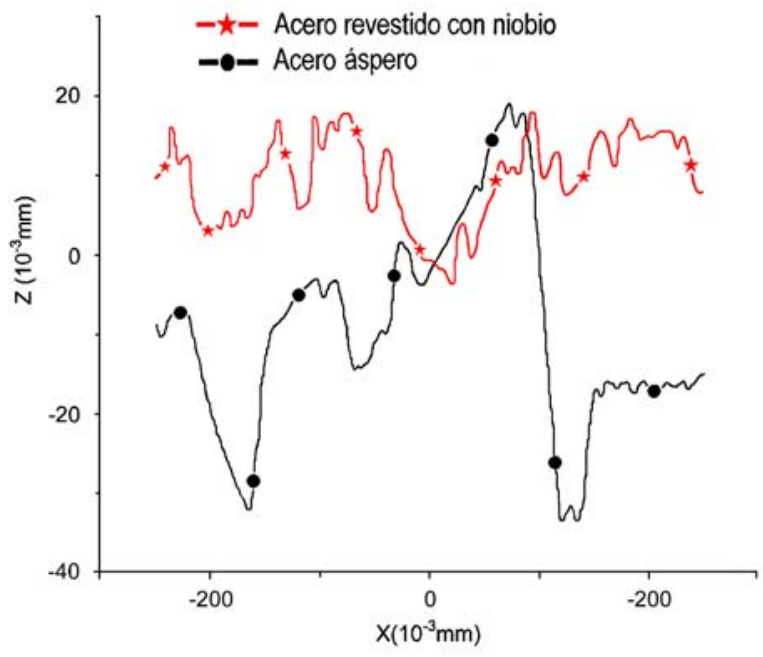

Fig. 4: Perfil de rugosidad superficial del acero pulido y revestido con niobio

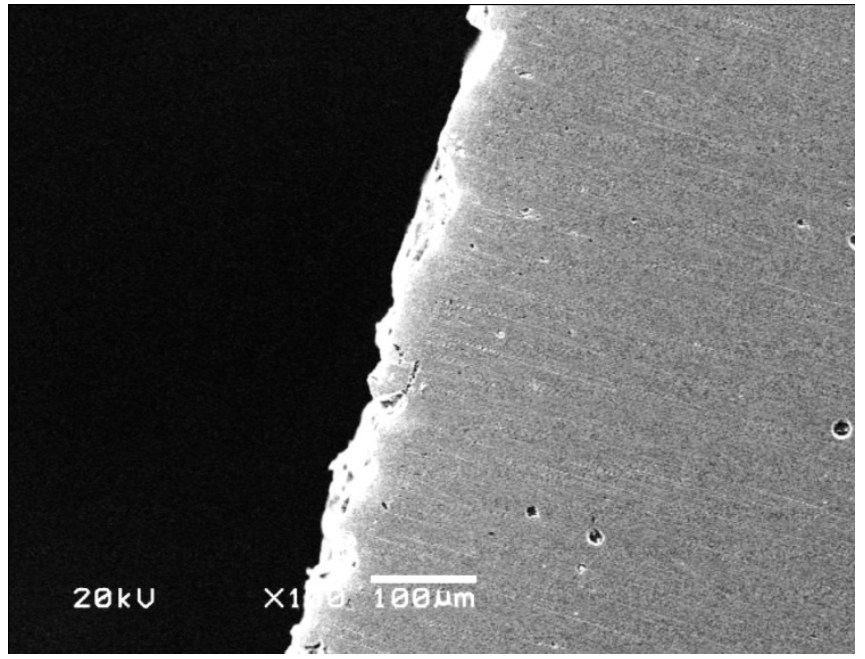

Fig. 5: Micrografía transversal de la superficie pulida obtenida por MEB

El valor de Ry inferior del que prevé la norma N-2568, puede haber influenciado negativamente para un buen anclaje del revestimiento. El ensayo de doblado a $180^{\circ} \mathrm{C}$ se realizó en un equipo MTS Servohydraulic (figura 6) identificándose primeramente el surgimiento de fisuras al inicio de la deformación plástica del revestimiento y un desplazamiento posterior de partes enteras del mismo (figura 7), por lo que este revestimiento no satisface los requisitos de adherencia. 


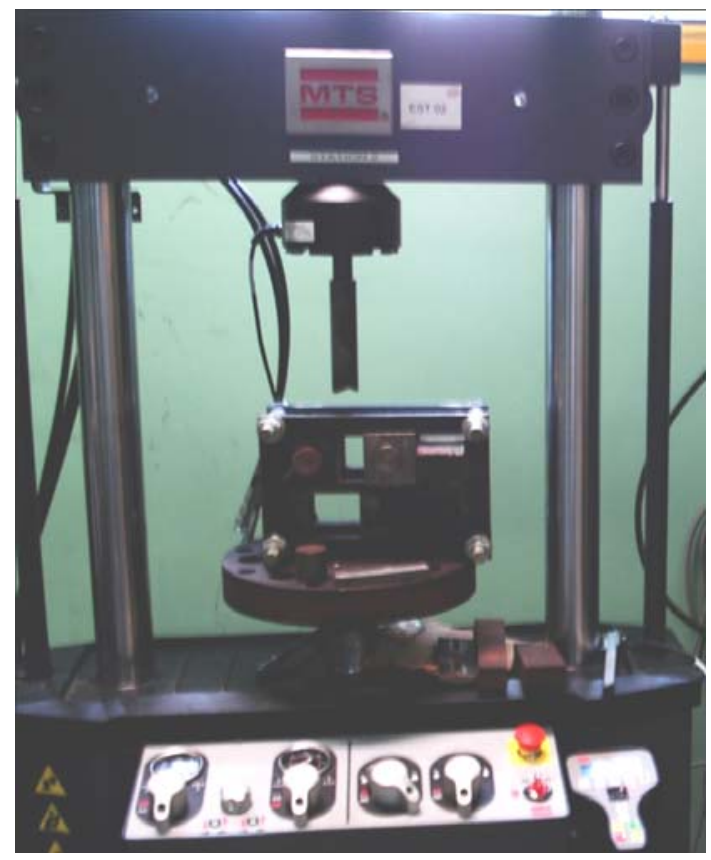

Fig. 6: Equipo de doblado MTS Servohydraulic

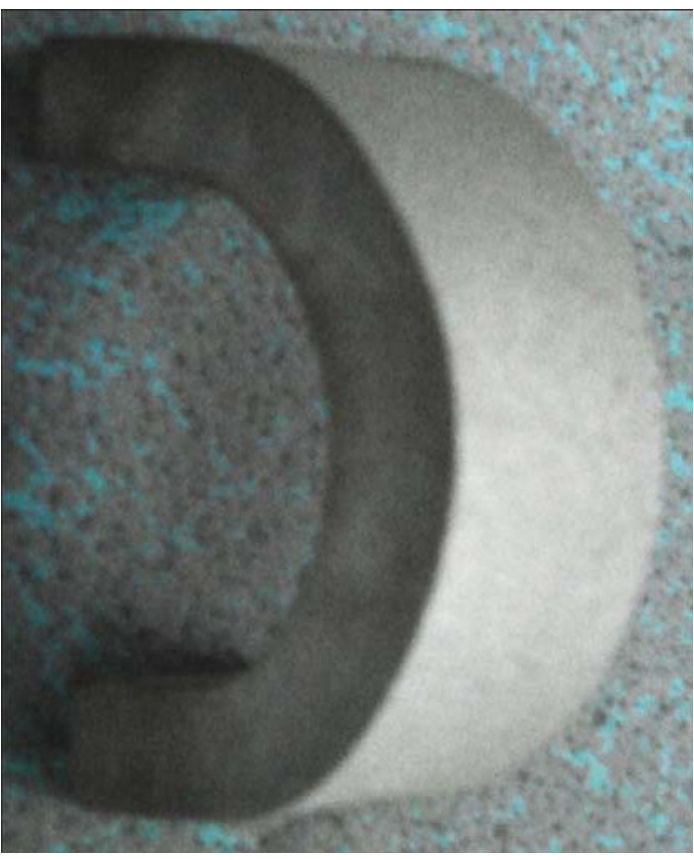

Fig. 7: Cuerpo de prueba reprobado luego del ensayo

A partir de las curvas de polarización obtenidas (figura 8) es posible observar el comportamiento electroquímico del substrato revestido con niobio comparativamente al mismo revestimiento. Se puede observar que la presencia del revestimiento disminuye las densidades de corriente desarrolladas por el sistema, comparativamente al acero revestido. Ese resultado muestra que aún presentando fallas el proceso actuó protegiendo al acero contra la corrosión.

Es posible observar a partir de las imágenes obtenidas por MEB (figura 3) que las fallas observadas (porosidades), no son pasantes es decir la presencia de los defectos observados no comprometió aparentemente la barrera establecida por el revestimiento entre el metal y el medio. También es preciso considerar que la falta de adherencia y la elevada rugosidad del revestimiento no contribuyen para un buen desempeño de ese revestimiento. De esa forma esos aspectos deben ser considerados en trabajos futuros buscando la optimización de las propiedades de revestimientos de niobio obtenidos por aspersión térmica.

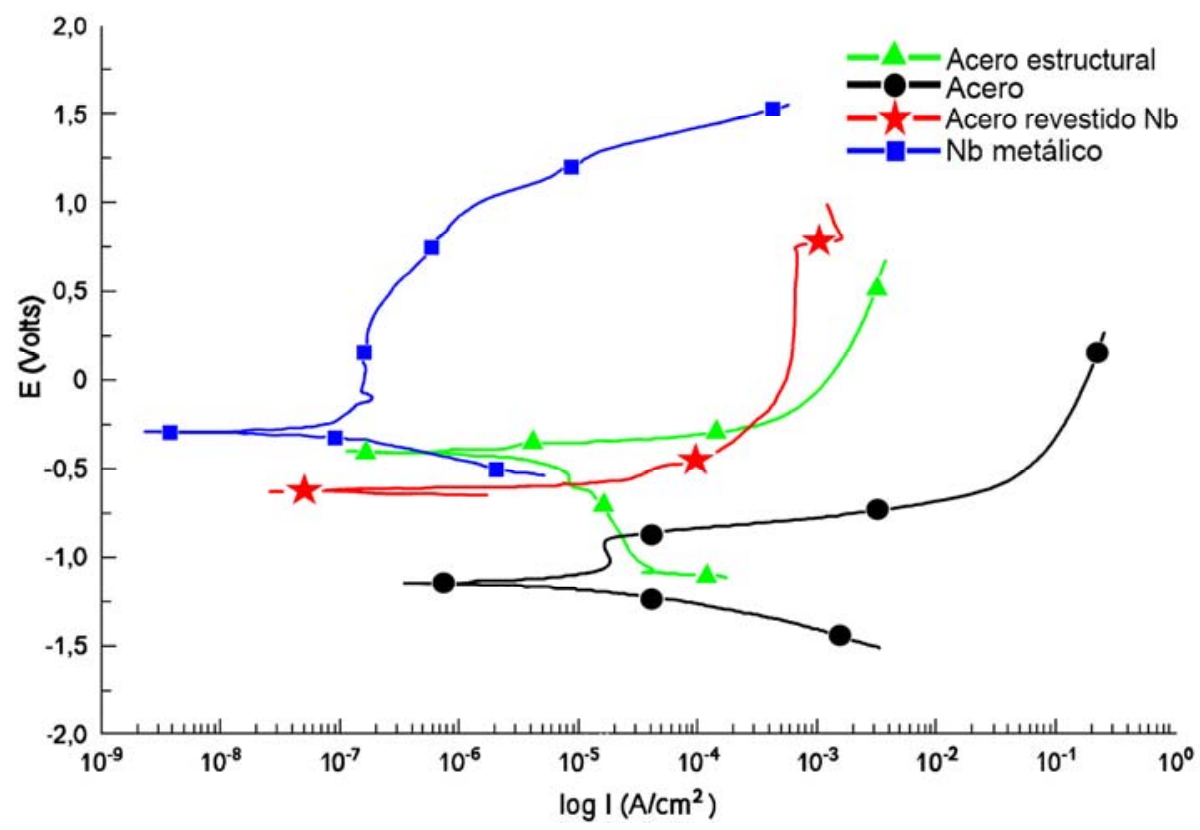

Fig. 8: Curva de polarización de los elementos estudiados, solución de $\mathrm{NaCl} 3,5 \%$ 


\section{CONCLUSIONES}

Los revestimientos obtenidos en el presente trabajo, presentan discontinuidades, como vacíos, fisuras y porosidades. Esto puede ser debido a los parámetros establecidos de la corriente eléctrica, granulometría del polvo de niobio y el flujo de gas de protección

La rugosidad superficial del acero pulido con alúmina quedo fuera del rango establecido por la norma, Petrobras N-2568, lo que contribuyó posiblemente a la falta de adherencia verificada en el ensayo de doblado.

Aunque el revestimiento presenta vacios y falta de llenado entre las placas esparcidas, fue posible observar que el revestimiento disminuyó los valores de la densidad de corriente desarrollados por el sistema comparativamente al acero no revestido, lo que le otorga mayor protección contra la corrosión.

\section{AGRADECIMIENTOS}

Los autores agradecen a las siguientes entidades por el apoyo prestado, Laboratorio de Investigaciones en Corrosión (LAPEC) de la Universidade do Rio Grande do Sul, al CNPq y la CAPES, que es la entidad del gobierno Brasilero orientado a la formación de recursos humanos.

\section{REFERENCIAS}

Ardelean, H., I. Frateur y P. Marcus, Corrosion protection of magnesium alloys by cerium, zirconium andniobium-based conversion coatings, Corrosion Science, 50, 1907-1918 (2008).

Carvalho, L.J, Estudo do comportamento de revestimentos a base de niobio em presença de petróleo com elevado índice de acidez, Tesis de magister, Dpto. Eng. Metalúrgica e de Materiais, UFRJ, Rio de Janeiro, Brasil (1998).

Carvalho, L.J, Estudo do comportamento de revestimentos à base de nióbio aplicados por aspersão térmica a chama na corrosão de aços ao carbono em presença de ácidos naftênicos e sulfetos em altas temperaturas, Tesis de doctorado, Dpto. Eng. Metalúrgica e de Materiais, UFRJ, Rio de Janeiro, Brasil (2004).

Chaves, N. W, Clad por explosão em câmara de vácuo. Seminário Interno Engenharia/Sequi, Petrobras, Rio de Janeiro, Brasil (2008).

Fenker, M., H. Kappl, P. Carvalho y F. Vaz, Thermal stability, mechanical and corrosion behaviour of niobium-based coatings in the ternary system Nb-O-N, The Solid Films, 519, 2457-2463 (2011).

Gentil V., Corrosão, pp 77-81, Livros técnicos e científicos Editora S.A, Rio de Janeiro, Brasil (1996)

Godoy, Y.C., E. A. Souza y G. M. P. Silva, Tensões residuais em conjugados processados por aspersão térmica e tratados termicamente, Revista Materiais, Belo Horizonte, Brasil (2007).

Hsieh, J.H., Niobium coating on 316L stainless stell for improving corrosion resistance, Surface and coating technology, 49(3), 83-86 (1991).

Lima, C.C. y R. Trevisan, Aspersão térmica fundamentos e aplicaçôes, Artliber Editora, São Paulo, Brasil (2001).

Majumdar, S., P. Sengupta, G. B. Kale y I. G. Sharma, Development of multilayer oxidation resistant coating on niobium and tantalum. Surface and coating technology, 200, 3713-3718 (2006). 
N-2568: Petrobras (Brasil), Revestimentos anti-corrosivos aplicados por aspersão térmica, 1-10, Rio de Janeiro-Brasil (2004).

Paredes, R., Revestimentos depositados por aspersão térmica utilizados na indústria do petróleo, $3^{\circ}$ seminário de materiais avançados, Curitiba, Brasil (2004).

Quelhas, K.A.S., Estudo da corrosão do niobio em médio metanólico, Tesis de doctorado, Dpto. Eng. Metalúrgica e de Materiais, UFRJ, Rio de Janeiro, Brasil (2007).

Thorpe, M.L., Thermal Spray, Advanced materials \& processes, 143(5), 50-61 (1993).

Zhang, L., D. Sun y H. Yu, Effect of niobium on the microstructure and wear resistance of ironbased alloy coating produced by plasma cladding. Materials Science and Engineering, 490, 57-61 (2008). 
